\title{
ON REAL QUADRATIC FIELDS
}

\section{GILLES LACHAUD}

1. Introduction. It is now well known that there are only nine imaginary quadratic fields $\mathbb{Q}(\sqrt{-d})$ with class number $h(-d)$ equal to one. We refer to the article of Stark (1969) for a detailed survey on this topic. This result has been previously obtained under a variant of the Riemann hypothesis (hypothesis (H) below). When one seeks for an analog of this result for real quadratic fields, it becomes clear that the hypotheses need to be adapted, indeed it is likely that there are an infinity of real quadratic fields with class number one. In place of the number $h(d)$, we consider here the number $\kappa(d)$ of reduced primitive quadratic forms of discriminant $d$, as explained below. One has $\kappa(d)=h(d)$ for $d<0$, but certainly not for $d>0$.

Let $K$ be a real quadratic field of discriminant $d>0$. A primitive binary integral quadratic form of discriminant $d$ is a polynomial

$$
Q(M, N)=A M^{2}+B M N+C N^{2} \in \mathbf{Z}[M, N]
$$

for which g.c. d. $(A, B, C)=1$ and $B^{2}-4 A C=d$. We write $Q=[A, B, C]$. Denote by $\mathbf{Q}(d)$ the set of primitive binary integral quadratic forms of discriminant $d$. The group $\Gamma_{0}=\operatorname{GL}(2, \mathbb{Z})$ operates on $\mathbf{Q}(d)$ and the quotient space $\Gamma_{0} \backslash \mathbf{Q}(d)$ is canonically isomorphic with the group $\mathbf{H}(d)$ of wide ideal classes of $K$. A form $Q=[A, B, C]$ is called reduced if it fulfills the conditions

$$
A>0, \quad B<0, \quad C<0, \quad|B|<\sqrt{d}, \quad \sqrt{d}-|B|<2 A<\sqrt{d}+|B| .
$$

The number $\kappa(d)$ of elements of the set $\mathbf{Q}_{\text {red }}(d)$ of reduced forms with discriminant $d$ will be called the caliber of the field $K=\mathbb{Q}(\sqrt{d})$. The conditions (R) imply $\operatorname{Max}(|A|,|B|,|C|)<\sqrt{d}$, hence the number $\kappa(d)$ is finite. Let $Q=[A, B, C] \in \mathbf{Q}(d)$, where $A>0$, and write

$$
Q(M, N)=A(M-N x)\left(M-N x^{\prime}\right),
$$

where we assume $x>x^{\prime}$. Write the continued fraction expansion of $x$ as

$$
x=\left[q_{0}, \ldots, q_{s-1}, r_{0}, \ldots, r_{m-1}, \ldots\right],
$$

with primitive period $\left(r_{0}, \ldots, r_{m-1}\right)$. When considered up to circular permutation, this primitive period is unchanged if we replace $Q$ by an equivalent form, and therefore only depends on the class $C$ of $Q$; the length $m(C)$ of this primitive period we call the caliber of the class $C$ (or of the form $Q$, or of the number $x$ ). If the form $Q$ is reduced, the reduced forms which are equivalent to $Q$ are exactly those which are obtained by a circular permutation of the

Received by the editors June 12, 1986 and, in revised form, November 30, 1986.

1980 Mathematics Subject Classification (1985 Revision). Primary 11E45, 11M41, 11R29, 11R42; Secondary 11E32, 11E41, 11M20, 11R11. 
period of the roots. Thus the number of elements in $\mathbf{Q}_{\text {red }}(d)$ of each class $C$ is equal to its caliber $m(C)$, hence:

$$
\kappa(d)=\sum_{C \in \mathbf{H}(d)} m(C) .
$$

2. General results. Let $\phi=(1+\sqrt{5}) / 2$ be the golden section, and let $\epsilon_{0}(d)$ be the fundamental unit of the field $K=\mathbb{Q}(\sqrt{d})$.

THEOREM 2.1. For every class $C$ of $K$, one has

$$
m(C) \log \phi \leq \log \epsilon_{0}(d)<m(C) \log \sqrt{d},
$$

and this result is best possible.

This theorem is classical; the first inequality is explicitly stated in Vijayaraghavan (1927). From these inequalities we obtain

$$
\kappa(d) \log \phi \leq h(d) \log \epsilon_{0}(d)<\kappa(d) \log \sqrt{d} ;
$$

since Siegel's theorem (1935) tells us that

$$
\log \left(h(d) \log \epsilon_{0}(d)\right) \sim \log \sqrt{d},
$$

we deduce

$$
\log \kappa(d) \sim \log \sqrt{d}
$$

and therefore:

THEOREM 2.2. There is only a finite number of real quadratic fields with a given caliber.

The estimate $(*)$ is not effective. But let us however introduce the hypothesis

$$
\varsigma(1 / 2, K) \leq 0 .
$$

Here $s(s, K)$ denotes the zeta function of $K$. Hypothesis $(\mathrm{H})$ clearly is a consequence of the Generalized Riemann Hypothesis on ]0,1[.

THEOREM 2.3. Under hypothesis $(\mathrm{H})$, one has

$$
h(d) \log \epsilon_{0}(d)<\omega_{1} \kappa(d),
$$

with $\omega_{1}<4.230 \ldots$

For $d \equiv 0(\bmod 4)$, Golubeva $(1984)$ has proven the following inequalities:

$$
\left(7 / \pi^{2}\right) \kappa(d)(1+o(1))<h(d) \log \epsilon_{0}(d)<\left(10 / \pi^{2}\right) \kappa(d)(1+o(1)),
$$

but her result is ineffective. From Theorem 2.3, making use of the trivial lower estimate $\epsilon_{0}(d)>(d-3)^{1 / 2}$, we deduce that

$$
d<\exp \left(2 \omega_{1} \kappa(d) / h(d)\right)+3 .
$$

3. Fields with caliber one. By definition, every field with caliber one is principal. If a reduced quadratic surd $x>1$ is of caliber one, then one has $x=r+(1 / x)$ with some $r \geq 1$, i.e., $x=(1 / 2)\left(r+\left(r^{2}+4\right)^{1 / 2}\right)$. Let $t$ be a 
squarefree natural integer. The principal class of $\mathbb{Q}(\sqrt{t})$ is of caliber one if and only if one of the following holds

$$
\begin{aligned}
& t=r^{2}+4 \quad \text { where } r \equiv 1(\bmod 4) \\
& 4 t=r^{2}+4 \quad \text { where } r \equiv 2(\bmod 4)
\end{aligned}
$$

the quadratic surd $x$ above is then the fundamental unit (with norm -1) of $\mathbb{Q}(\sqrt{t})$; these facts go back at least to Richaud (1866). From Theorem 2.3 we deduce

THEOREM 3.1. Under hypothesis $(\mathrm{H})$, the only real quadratic fields with caliber one are the seven fields $\mathbb{Q}(\sqrt{t})$ with $t=2,5,13,29,53,173,293$.

In the same vein, one also has

THEOREM 3.2. Under hypothesis $(\mathrm{H})$, the only principal real quadratic fields with $d=r^{2}+1 \equiv 1(\bmod 4)$ are the six fields $\mathbb{Q}(\sqrt{t})$ with $t=5,17$, $37,101,197,677$.

This theorem has been the subject of a question of Chowla for many years: cf. e.g. Chowla and Friedlander (1976). The fields considered in Theorem 3.2 have caliber 3 , but they contain an order with caliber one. Çallialp (1980) has proven that hypothesis $(\mathrm{H})$ is satisfied for the real quadratic fields with discriminant $d=r^{2}+1 \equiv 1(\bmod 16)$. We thus have the following unconditional result: The only principal real quadratic field with discriminant $d=r^{2}+1 \equiv 1$ (mod 16$)$ is the field $\mathbb{Q}(\sqrt{17})$. However, one can prove this easily, using the fact that 2 splits in such a field.

4. An analytical result. We now look at the zeta functions of classes with small caliber. Let $\left(K_{i}\right)$ be an infinite sequence of real quadratic fields, set $d_{i}=\operatorname{disc}\left(K_{i}\right)$. Let $C_{i}$ be an ideal class of $K_{i}$ with caliber $m_{i}$. Assume moreover that $\log m_{i} \sim q \log d_{i}$ with $q \in[0,1 / 2[$. Let $A>0$.

THEOREM 4.1. If $d_{i}$ is sufficiently large, the partial zeta function $\varsigma\left(s, C_{i}\right)$ of the class $C_{i}$ has exactly one zero $s_{i}$ in the interval $\left[1-\left(A / \log d_{i}\right), 1\right]$; if $s_{i}=1-T_{i}^{-1}$, then

$$
\log T_{i} \sim((1 / 2)-q) \log d_{i} .
$$

In other words, the first part of the theorem says that the partial zeta function $\varsigma\left(s, C_{i}\right)$ does have a Siegel zero.

5. The method: A formula of Hecke and $H$-functions. For $Q=$ $[A, B, C]$ in $\mathbf{Q}(d)$, let $H_{Q}$ be the upper half-circle in the Poincaré upper halfplane $H$ whose equation is

$$
A\left(X^{2}+Y^{2}\right)+B X+C=0 .
$$

Let $\Gamma=\mathrm{SL}(2, \mathbb{Z})$, and let $\Gamma_{Q}$ be the subgroup of proper integral automorphisms of the form $Q$. Set $X=\Gamma \backslash H$ and $X_{Q}=\Gamma_{Q} \backslash H_{Q}$. The curve $X_{Q}$ is a closed primitive geodesic of $X$, and all the closed primitive geodesics of $X$ 
are of this form. For $\operatorname{Re}(s)>1$ we define

$$
\begin{aligned}
& Z^{*}(s, Q)=\pi^{-s} d^{s / 2} \Gamma(s / 2)^{2} \sum_{(m, n) \in \mathbb{Z}^{2} / \Gamma_{Q}} Q(m, n)^{-s}, \\
& E^{*}(z, s)=\pi^{-s} \Gamma(s) \varsigma(2 s) \sum_{(m, n)=1}(\operatorname{Im} z)^{s} /|m z+n|^{2 s} .
\end{aligned}
$$

The functions $E^{*}(z, s)$ are the usual Eisenstein series. They admit a meromorphic continuation to the complex plane, with poles at $s=0$ and $s=1$. They are invariant under $s \rightarrow 1-s$. A formula of Hecke states: for $s \neq 0,1$

$$
Z^{*}(s, Q)=\int_{X_{Q}} E^{*}(z, s)\left|d_{Q} z\right|
$$

for a suitable differential $d_{Q} z$. We refer to Hecke (1917), Lang (1970), Zagier (1975), Wielonsky (1984) for several proofs and applications of this equation. If $x$ and $x^{\prime}\left(x>x^{\prime}\right)$ are the roots of $Q$, a parametric representation of $H_{Q}$ is given by $\varphi(\lambda)=\left(x+i \lambda x^{\prime}\right) /(1+i \lambda)(0<\lambda<\infty)$. Let $x_{1}=1 /(x-[x])$ and set

$$
J_{Q}=\left\{\varphi(\lambda)|| x_{1}^{\prime} \mid<\lambda \leq x\right\} \subset H_{Q} .
$$

Let $Y_{Q}$ be the image of the path $J_{Q}$ in $X_{Q}$. For $s \neq 0,1$ define

$$
H^{*}(s, Q)=\int_{Y_{Q}} E^{*}(z, s)\left|d_{Q} z\right|
$$

and let $H(s, Q)$ be the function such that

$$
H^{*}(s, Q)=\pi^{-s} d^{s / 2} \Gamma(s / 2)^{2} H(s, Q) \text {. }
$$

If the primitive period of $x$ is $\left(x_{0}, \ldots, x_{m-1}\right)$, we set $Y_{n}=Y_{Q}$ for

$$
Q(M, N)=\left(M-N x_{n}\right)\left(M-N x_{n}^{\prime}\right) \quad(0 \leq n \leq m-1) .
$$

Proposition 5.1. The geodesic $X_{Q}$ is the disjoint union of the paths $Y_{n}$, for $0 \leq n \leq m-1$, and

$$
Z(s, Q)=\sum_{0 \leq n \leq m-1} H\left(s, Q_{n}\right) .
$$

We have thus obtained a decomposition of the function $Z(s, Q)$ as a sum of functions $H\left(s, Q_{n}\right)$ indexed by the primitive period of $Q$; this decomposition relies on the same principles as those of Shintani (1976) and Zagier (1977), but here the functions $H(s, Q)$ are no longer Dirichlet series. Using the asymptotic expansion of Eisenstein series at $s=1 / 2$ (cf. Chowla-Selberg (1967)), and the fact that $\operatorname{Im}(z) \geq 1 / 2$ for $z \in Y_{Q}$, we obtain the following result.

Proposition 5.2. Let $\gamma$ be the Euler constant and let

$$
\omega=\pi / 2+\log 8 \pi-\gamma=4.217 \ldots ;
$$

let $k=k_{Q}=\sqrt{d} / a_{Q}$. We then have

$$
d^{1 / 2} H(1 / 2, Q)=k^{1 / 2}(\log k-\omega)+R(Q),
$$

with $-1 / 50 \leq R(Q) \leq 1$. In particular if $k_{Q}>69$ then $H(1 / 2, Q)>0$.

Proposition 5.2 immediately implies Theorem 3.1: Indeed, if $K$ is a field with caliber one and if $Q$ is the principal form of $K$, one has

$$
H(s, Q)=Z(s, Q)=\varsigma_{K}(s) \text {. }
$$


Theorem 2.3 also follows from Proposition 5.2 and from the standard convexity inequalities; one can take $\omega_{1}=\omega+(1 / 80)$ in these theorems. Theorem 4.1 is proved using an "asymptotic limit formula." This formula is obtained from Hecke's formula when $s \rightarrow 1$, in much the same spirit as in the work of Goldfeld (1976); see also Goldfeld-Schinzel (1975).

\section{REFERENCES}

F. Callialp, Non-nullité des fonctions zêta des corps quadratiques réels pour $0<s<1$, C. R. Acad. Sci. Paris 291 (1980), 623-625.

S. Chowla and J. B. Friedlander, Some remarks on L-functions and class numbers, Acta Arith. 28 (1976), 414-417.

S. Chowla and A. Selberg, On Epstein's zeta-function, J. Reine Angew. Math. 227 (1967), 86-110.

E. P. Golubeva, On the length of the period of a quadratic irrationality, Mat. Sb. 123 (1984), no. 1; English transl. in Math. USSR-Sbornik 51 (1985), 119-128.

D. Goldfeld and A. Schinzel, On Siegel's zero, Ann. Sci. Norm. Sup. Pisa IV 2 (1975), 571-585.

D. Goldfeld, The class number of quadratic fields and the conjectures of Birch and Swinnerton-Dyer, Ann. Sci. Norm. Sup. Pisa IV 3 (1976), 623-663.

E. Hecke, Über die Kroneckersche grenzformel für reelle quadratische körper und die classenzahl relativ- abelscher körper, Mathematische Werke, Vandenhoeck und Ruprecht, Göttingen, 1970; no. 10, 1917.

S. Lang, Algebraic number theory, Addison-Wesley, Reading, 1970.

Casimir Richaud, Sur la résolution des équations $x^{2}-A y^{2}= \pm 1$, Atti dell'Acc. Pont. de Nuovi Lincei (1866), 177-182.

T. Shintani, On evaluation of zeta functions of totally real algebraic number fields at nonpositive integers, J. Fac. Sci. Univ. Tokyo, Sect. IA 23 (1976), 393-417.

C. L. Siegel, Über die Classenzahl quadratischer Zahlkörper, Acta Arith. 1 (1935), 83-86; =Ges. Abh., no. 21, I, pp. 406-409, Springer, Berlin, 1966.

H. Stark, On the problem of unique factorization in complex quadratic fields, Proc. Sympos. Pure Math., vol. 12, Amer. Math. Soc., Providence, R.I., 1969, pp. 41-56.

T. Vijayaraghavan, Periodic simple continued fractions, Proc. London Math. Soc. 26 (1927), 403-414.

F. Wielonsky, Séries d'Eisenstein, intégrales torö̈dales et une formule de Hecke, Enseign. Math. 31 (1985), 93-135.

D. Zagier, A Kronecker limit formula for real quadratic fields, Math. Ann. 213 (1975), 153-184.

, Valeurs des fonctions zêta des corps quadratiques réels aux entiers négatifs, J. Arith. Caen, Astérisque 41-42 (1977), 135-151.

C.I.R.M., CASE 916, Luminy, 13288 Marseille CedeX 9, France 